\title{
Hipoadrenocorticismo primário em um cão
}

\author{
Primary hipoadrenocorticism in a dog
}

\section{Mauren Picada Emanuelli ${ }^{\mathrm{I}^{*}}$ Sonia Terezinha dos Anjos Lopes ${ }^{\text {II }}$ Claudete Schmidt ${ }^{\text {II }}$ Roberto Marinho Maciel ${ }^{\mathrm{I}}$ Carmen Lice Buchmann de Godoy ${ }^{\text {III }}$}

\begin{abstract}
O presente relato descreve um caso de hipoadrenocorticismo primário em uma fêmea canina, com seis anos de idade, sem raça definida, atendida no Hospital de Clínicas Veterinárias da Universidade Federal de Santa Maria. O cão apresentava letargia, vômito, anorexia, fraqueza, tremores, poliúria e polidipsia. Há um mês o animal estava sendo tratado para insuficiência renal crônica sem melhora clínica. Nos exames laboratoriais, evidenciaram-se anemia normocítica normocrômica, azotemia, hiponatremia, hipercalemia, hipercalcemia e hiperfosfatemia e, por meio de radiografia, foi observada microcardia. O diagnóstico foi confirmado pelas alterações eletrolíticas, principalmente por diminuição da relação sódio:potássio e pelo teste de estimulação com corticotrofina, que resultou em baixa concentração plasmática de cortisol pós-estimulação. Após o diagnóstico, iniciou-se terapia com acetato de fludrocortisona, com melhora significativa dos sinais clínicos depois de dois meses de tratamento.
\end{abstract}

Palavras-chave: hipoadrenocorticismo, mineralocorticóides, glicocorticóides, cães.

\section{ABSTRACT}

This study describes a primary hypoadrenocorticism in a mixed-breed six years old bitch examined at the Federal University of Santa Maria Veterinary Hospital. The animal presented lethargy, vomiting, anorexia, weakness, shaking, polyuria and polydipsia. During a month the animal was medicated for chronic renal failure, showing no clinical progress. Laboratory exams evidenced a normocytic normochromic anemia, azotemia, hyponatremia, hyperkalemia, hypercalcemia and hyperphosphatemia. Microcardia was observed on radiographic exam. The diagnosis was confirmed by altered electrolytic pattern, mainly by sodium:potassium ratio and by corticotrophin stimulation test, which resulted in low plasma cortisol. After the diagnosis fludrocortisone acetate therapy has begun, showing a clinical evolution after two months treatment.

Key words: hypoadrenocorticism, mineralocorticoids, glucocorticoids, dogs.

O hipoadrenocorticismo é uma doença endócrina pouco comum em cães, resultado da produção adrenal deficiente de mineralocorticóide e/ ou glicocorticóide. A destruição do córtex adrenal caracteriza o hipoadrenocorticismo primário (doença de Addison), que é a causa mais comum da doença em cães. Esta destruição pode resultar de várias causas; entretanto, um distúrbio auto-imune ou idiopático parece ser o principal fator (SCHAER et al., 1986). O hipoadrenocorticismo secundário é uma condição rara que se caracteriza pela secreção hipofisária deficiente do hormônio adrenocorticotrópico (ACTH) (FELDMAN \& TYRRELL, 1977; REUSCH, 2004). A doença geralmente acomete cães de meia-idade, embora já tenha sido relatada em animais de quatro meses até

IPrograma de Pós-graduação em Medicina Veterinária, Universidade Federal de Santa Maria (UFSM), Santa Maria, RS, Brasil. *Endereço para correspondência: Rua José Crivelaro, 215, apt 201, km 3, 97095-330, Santa Maria, RS, Brasil. E-mail: maurenvet@hotmail.com.

IIDepartamento de Clínica de Pequenos Animais, UFSM, Santa Maria, RS, Brasil.

IIIDepartamento de Grandes Animais, UFSM, Santa Maria, RS, Brasil. 
12 anos de idade. As fêmeas são mais acometidas que os machos, sendo aproximadamente $70 \%$ dos casos de insuficiência adrenocortical idiopática de ocorrência em cadelas (FELDMAN \& PETERSON, 1984; MELIÁN \& PETERSON, 1996).

No hipoadrenocorticismo primário, a maioria das manifestações clínicas é atribuída à deficiência de aldosterona e cortisol. Na insuficiência adrenal secundária, a deficiência na secreção do ACTH resulta, na maioria das vezes, apenas em inadequada produção de glicocorticóide, sem alterar a produção de mineralocorticóide (KINTZER \& PETERSON, 1997).

A deficiência de cortisol leva a letargia, fraqueza, tolerância reduzida ao estresse e a várias alterações gastrintestinais, como anorexia, vômito, diarréia, dor abdominal e perda de peso (FELDMAN \& PETERSON, 1984).

A aldosterona é responsável pela absorção de sódio e excreção de potássio pelos rins. Com a falta de aldosterona, a concentração de íons potássio no líquido extracelular aumenta muito, levando à rápida perda de sódio, cloreto e água. Com isso o volume total do líquido extracelular e o volume sanguíneo ficam reduzidos (GUYTON \& HALL, 2002). Ocorre hipovolemia com diminuição do débito cardíaco e conseqüente redução da perfusão renal e de outros tecidos. A diminuição da filtração glomerular leva a azotemia pré-renal. A taxa de filtração glomerular reduzida e a diminuição da troca de sódio também levam a hipercalemia, que resulta em fraqueza muscular generalizada, excitabilidade miocárdica reduzida e nível refratário miocárdico aumentado (FELDMAN \& PETERSON, 1984; REUSCH, 2004).

Em um estudo realizado com 42 cães, MELIÁN \& PETERSON (1996) relataram como sinais clínicos mais comuns perda de apetite, letargia e vômito. Perda de peso, tremores, diarréia, poliúria/polidipsia e curso de aparecimento e desaparecimento da doença também são freqüentes. Isto pode ser explicado pela lenta destruição do córtex adrenal, que ainda pode ser capaz de responder às demandas normais, mas não às situações de estresse. Ao exame físico, os achados mais comuns são depressão, fraqueza, síncope, hipotermia e desidratação. FELDMAN \& PETERSON (1984) ainda citam a ocorrência de bradicardia, pulso femoral fraco, edema e melena como achados menos freqüentes.

Os achados laboratoriais incluem anemia normocítica normocrômica, eosinofilia e linfocitose absoluta (FELDMAN \& FELDMAN, 1977; MELIÁN \& PETERSON, 1996). Azotemia pré-renal com aumento da uréia e creatinina sanguínea e hiperfosfatemia ocorrem na maioria dos cães (MELIÁN \& PETERSON,
1996). A densidade urinária pode estar baixa (<1030), mas não tão baixa quando comparada àquela encontrada em animais com doença renal primária (FELDMAN \& FELDMAN, 1977). Hipoglicemia (FELDMAN \& PETERSON, 1984), hipoalbuminemia (LANGLAIS-BURGESS el al., 1995) e hipercalcemia (PETERSON \& FEINMAN, 1982) também podem estar presentes. Outros achados são: aumento dos níveis de alanina aminotransferase (ALT), de aspartato aminotransferase (AST) e, menos freqüentemente, da fosfatase alcalina (FA) (PETERSON et al., 1996). Acidose metabólica freqüentemente é observada (REUSCH, 2004).

As alterações eletrolíticas incluem hiponatremia, hipocloremia e hipercalemia. A redução na relação sódio:potássio ( $\mathrm{Na}: \mathrm{K})$, que normalmente varia de 27:1 a 40:1, é um dos achados laboratoriais clássicos de hipoadrenocorticismo primário (PETERSON et al., 1996).

Os achados radiográficos incluem microcardia, redução do lúmen da veia cava caudal, hipoperfusão dos campos pulmonares e fígado diminuído (MELIÁN et al., 1999). A ultra-sonografia pode revelar redução significativa no comprimento e na espessura das adrenais, se comparadas à de cães saudáveis (HOERAUF \& REUSCH, 1999). No eletrocardiograma, podem-se evidenciar alterações no traçado devido à hipercalemia, que deprime a condução cardíaca (FELDMAN \& PETERSON, 1984).

O diagnóstico de hipoadrenocorticismo pode ser feito pelo teste de estimulação com ACTH, que consiste na determinação do cortisol plasmático antes e uma hora após a administração intramuscular ou intravenosa de $0,25 \mathrm{mg}$ de ACTH sintético, ou antes e duas horas após a administração intramuscular de 2,2UI kg-1 de ACTH gel. Uma concentração do cortisol plasmático igual ou menor que $2 \mu \mathrm{g} \mathrm{dL}^{-1}$ após a estimulação com ACTH confirma o diagnóstico. Entretanto, em casos de destruição incompleta do córtex adrenal ou no hipoadrenocorticismo secundário, a concentração pode ser maior que $2 \mu \mathrm{g} \mathrm{dL} \mathrm{dL}^{-1}$. Este teste não diferencia o hipoadrenocorticismo primário do secundário; para isso, é necessário determinar-se a concentração do ACTH plasmático endógeno (FELDMAN \& PETERSON, 1984; REUSCH, 2004).

Os cães que apresentam alterações nas concentrações eletrolíticas, como diminuição na relação Na:K e secreção de cortisol anormalmente reduzida após estimulação com ACTH, podem ser diagnosticados como portadores de hipoadrenocorticismo primário, enquanto aqueles com eletrólitos dentro dos valores de referência devem ser investigados para hipoadrenocorticismo 
secundário(FELDMAN \& PETERSON, 1984; REUSCH, 2004).

O tratamento consiste em restabelecer a volemia, principalmente na crise adrenal aguda, pela administração de fluidoterapia intravenosa, de preferência de solução de cloreto de sódio 0,9\% e glicocorticóide. Este procedimento melhora a condição do animal na medida em que restaura a perfusão renal, o que geralmente é suficiente para estabilizar o paciente. No hipoadrenocorticismo primário, ainda é necessária a administração de mineralocorticóide para a manutenção do equilíbrio eletrolítico. Em qualquer dos casos de hipoadrenocorticismo, o paciente deve ser medicado ininterruptamente e monitorado periodicamente, o que favorece a manutenção de uma vida normal e de melhor qualidade. Como mineralocorticóide, está disponível no Brasil o acetato de fludrocortisona, que também tem atividade glicocorticóide. O pivalato de desoxicorticosterona (DOCP) tem atividade quase exclusivamente mineralocorticóide, mas não está disponível no Brasil (REUSCH, 2004).

De forma a contribuir para o diagnóstico da doença, o objetivo deste trabalho foi relatar os sinais clínicos, as alterações hematológicas, eletrolíticas e radiológicas que caracterizam o hipoadrenocorticismo primário em cães.

O presente relato descreve um caso de hipoadrenocorticismo primário em uma fêmea canina, com seis anos de idade, sem raça definida, atendida no Hospital de Clínicas Veterinárias da Universidade Federal de Santa Maria. O cão apresentava letargia, vômito, anorexia, fraqueza, tremores, poliúria e polidipsia. Há um mês o animal estava sob tratamento clínico conservador para insuficiência renal crônica, com orientações para uma dieta com diminuição de proteína, sódio, fósforo e suplemento de vitaminas do complexo B, no entanto, sem melhora clínica.

Neste caso, observa-se que o sexo e a idade do animal estão de acordo com FELDMAN \& PETERSON (1984) e MELIÁN \& PETERSON (1996), que citam uma predisposição maior da doença em fêmeas de meia-idade. Os sinais clínicos apresentados, apesar de inespecíficos e compatíveis com outros distúrbios, como doenças gastrintestinais e renais, são os mesmos relatados por MELIÁN \& PETERSON (1996): apatia, vômito, anorexia, fraqueza e tremores que ocorrem pela deficiência de glicocorticóide. A poliúria, secundária à perda urinária de sódio com polidipsia compensatória, ocorre devido à deficiência de mineralocorticóide.

Nos exames laboratoriais, evidenciou-se anemia normocítica normocrômica, azotemia, hiponatremia, hipercalemia, hipercalcemia e hiperfosfatemia. O hemograma foi feito em aparelho automático PENTRA ${ }^{\circledR}$, a mensuração de sódio, potássio e cloretos com a metodologia de química secapotenciometria, e cálcio, fósforo, creatinina e uréia por química seca-colorimétrico, em equipamento VITROS ${ }^{\circledR}$ 950 Chemistry System.

Segundo LIFTON et al. (1996), a anemia não regenerativa, confirmada pelo número de eritrócitos de 3,21uL ${ }^{-1}$, VCM de 74,76fL e CHCM de 33,33\%, resulta da supressão da medula óssea pela deficiência de glicocorticóides. A azotemia pré-renal ocorre devido à hipovolemia e à conseqüente hipoperfusão renal, como descrito por MELIÁN \& PETERSON (1996), sendo confirmada, neste caso, pelo aumento na creatinina (7,8mg dL $\left.{ }^{-1}\right)$ e na uréia (298mg dL $\left.\mathrm{dL}^{-1}\right)$ séricas. Segundo PETERSON et al. (1996), a hipercalemia e a hiponatremia são os achados laboratoriais clássicos de hipoadrenocorticismo primário devido à deficiência de aldosterona, que interfere na capacidade dos rins em excretar potássio e absorver sódio. Fisiologicamente, a relação Na:K não deve ser inferior a 27:1. No presente relato, o paciente apresentava valores de sódio de $130 \mathrm{mmol} \mathrm{L}^{-1}$ e potássio de $8,3 \mathrm{mmol} \mathrm{L}^{-1}$, sendo a relação Na:K de 15,66:1, sugerindo uma possível deficiência de mineralocorticóide. A hipercalcemia (12,5 $\left.\mathrm{mg} \mathrm{dL}^{-1}\right)$ e a hiperfosfatemia $\left(11,1 \mathrm{mg} \mathrm{dL}^{-1}\right)$ presentes neste animal parecem ocorrer devido à diminuição na filtração glomerular, que resulta em baixa excreção renal de cálcio e fósforo (REUSCH, 2004).

Por meio de radiografia convencional, foi observada microcardia, que é um dos achados radiográficos descritos por MELIÁN et al. (1999) como de ocorrência em 50\% dos cães com hipoadrenocorticismo, e está relacionada ao grau de hipovolemia (REUSCH, 2004).

Foi realizado o teste de estimulação com ACTH sintético ${ }^{a}$, na dose de $0,25 \mathrm{mg}$, administrada pela via intramuscular. Foram coletadas amostras de sangue antes e uma hora após a aplicação do ACTH. A dosagem do cortisol sérico foi realizada pelo método de radioimunoensaio em contador GAMA. O teste teve como resultados $0,3 \mu \mathrm{g} \mathrm{dL} \mathrm{d}^{-1}$ de cortisol plasmático pré ACTH e $0,44 \mu g \mathrm{dL}^{-1}$ pós-ACTH, o que, segundo REUSCH (2004), confirma o diagnóstico de hipoadrenocorticismo. A presença das alterações eletrolíticas, principalmente de diminuição da relação $\mathrm{Na}$ K, teve grande valor na diferenciação do hipoadrenocorticismo primário do secundário, uma vez que, segundo KINTZER \& PETERSON (1997), no hipoadrenocorticismo secundário, não há deficiência de aldosterona e, por isso, as alterações eletrolíticas não estão presentes.

Ciência Rural, v.37, n.5, set-out, 2007. 
O restabelecimento da volemia foi feito por fluidoterapia intravenosa com $1,5 \mathrm{~L}$ de solução de cloreto de sódio $0,9 \%$, diariamente, e hemissuccinato de hidrocortisona ${ }^{\mathrm{b}}$ na dose de $0,6 \mathrm{mg} \mathrm{kg}^{-1}$ por dia, conforme descrito por FERGUSON \& HOENIG (2003), até a confirmação do diagnóstico pelo teste de estimulação com ACTH. Após a instauração do diagnóstico definitivo, iniciou-se terapia com acetato de fludrocortisona ${ }^{\mathrm{c}}$ na dose de $0,015 \mathrm{mg} \mathrm{kg}^{-1} \mathrm{dia}^{-1}$, em doses divididas com 12 horas de intervalo, seguindo um dos protocolos descritos por REUSCH (2004) para o tratamento de hipoadrenocorticismo primário.

Após dois meses do início da terapia, os valores do hemograma normalizaram-se e a relação $\mathrm{Na}: \mathrm{K}$ aumentou para 26,6:1, valor este muito próximo aos valores normais. No quarto mês de tratamento, o exame radiográfico mostrou tamanho normal do coração, como resultado do restabelecimento da volemia, e os perfis bioquímico, eletrolítico e hematológico encontravamse sem alterações, o que reflete o controle da doença.

A descrição deste caso traz informações importantes aos clínicos para diagnosticar esta doença. Os exames complementares são de extrema importância para diferenciar o hipoadrenocorticismo primário do secundário e, desta maneira, juntamente com o exame específico, chegar-se ao diagnóstico.

\section{FONTESDEAQUISIÇÃO}

asynacthène, Novartis, Rueil-Malmaison.

'Solu-cortef, Rhodia, São Paulo.

cFlorinefe, Bristol-Myers, São Paulo.

\section{REFERÊNCIAS}

FELDMAN, B.F.; FELDMAN, E.C. Routine laboratory abnormalities in endocrine disease. Vet Clin North Am: Small Anim Pract, v.7, n.3, p.443-464, 1977.

FELDMAN, E.C.; PETERSON, M.E. Hypoadrenocorticism. Vet Clin North Am: Small Anim Pract, v.14, n.4, p.751766, 1984.
FELDMAN, E.C.; TYRRELL, J.B. Hypoadrenocorticism. Vet Clin North Am: Small Anim Pract, v.7, n.3, p.555-581, 1977.

FERGUSON, D.C; HOENIG, M. Glicocorticóides, mineralocorticóides e inibidores da síntese de esteróides. In: ADAMS, H.R. Farmacologia e terapêutica em veterinária. 8.ed. Rio de Janeiro: Guanabara-Koogan, 2003. p.542-561.

GUYTON, A.C.; HALL, J.E. Os hormônios adrenocorticais. In: _ Tratado de fisiologia médica. 10.ed. Rio de Janeiro: Guanabara-Koogan, 2002. Cap.77, p.813-826.

HOERAUF, A.; REUSCH, C. Ultrasonographic evaluation of the adrenal glands in six dogs with hypoadrenocorticism. J Am Anim Hosp Assoc, v.35, n.3, p.214-218, 1999.

KINTZER, P.P. PETERSON, M.E. Primary and secondary canine hypoadrenocorticism. Vet Clin North Am: Small Anim Pract, v.27, n.2, p.349-357, 1997.

LANGLAIS-BURGESS, L. et al. Concurrent hypoadrenocorticism and hypoalbuminemia in dogs: a retrospective study. J Am Vet Hosp Assoc, v.31, n.4, p.307-311, 1995.

LIFTON, S.J. et al. Glucocorticoid deficient hypoadrenocorticism in dogs: 18 cases (1986-1995). J Am Vet Med Assoc, v.209, n.12, p.2076-2081, 1996.

MELIÁN, C. PETERSON, M.E. Diagnosis and treatment of naturally occurring hypoadrenocorticism in 42 dogs. J Small Anim Pract, v.37, n.6, p.268-275, 1996.

MELIÁN, C. et al. Radiographic findings in dogs with naturallyoccurring primary hypoadrenocorticism. J Am Anim Hosp Assoc, v.35, n.3, p.208-212, 1999.

PETERSON, M.E. et al. Pretreatment clinical and laboratory findings in dog with hypoadrenocorticism: 225 cases (19791993). J Am Vet Med Assoc, v.208, n.1, p.85-91, 1996.

PETERSON, M.E.; FEINMAN, J.M. Hypercalcemia associated with hypoadrenocorticism in sixteen dogs. J Am Vet Med Assoc, v.181, n.8, p.802-804, 1982.

REUSCH, C.E. Hipoadrenocorticismo. In: ETTINGER, S.J.; FELDMAN, E.C. Tratado de medicina interna veterinária - doenças do cão e do gato. 5.ed. Rio de Janeiro: GuanabaraKoogan, 2004. V.2, p.1569-1580.

SCHAER, M. et al. Autoimmunity and Addison's disease in the dog. J Am Anim Hosp Assoc, v.22, n.6, p.789-794, 1986. 